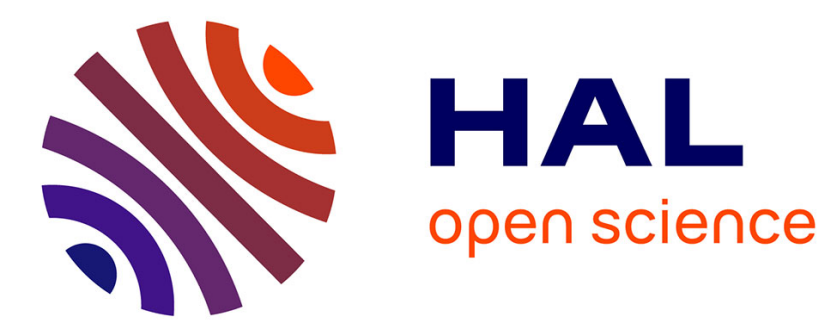

\title{
Short range order in Ge-Ga-Se glasses
}

I. Pethes, R. Chahal, Virginie Nazabal, C. Prestipino, A. Trapananti, C.

Pantalei, B. Beuneu, B. Bureau, P Jóvári

\section{To cite this version:}

I. Pethes, R. Chahal, Virginie Nazabal, C. Prestipino, A. Trapananti, et al.. Short range order in Ge-Ga-Se glasses. Journal of Alloys and Compounds, 2015, 651, pp.578-584. 10.1016/j.jallcom.2015.08.039 . hal-01188226

HAL Id: hal-01188226

\section{https://hal-univ-rennes1.archives-ouvertes.fr/hal-01188226}

Submitted on 25 Nov 2015

HAL is a multi-disciplinary open access archive for the deposit and dissemination of scientific research documents, whether they are published or not. The documents may come from teaching and research institutions in France or abroad, or from public or private research centers.
L'archive ouverte pluridisciplinaire HAL, est destinée au dépôt et à la diffusion de documents scientifiques de niveau recherche, publiés ou non, émanant des établissements d'enseignement et de recherche français ou étrangers, des laboratoires publics ou privés. 


\title{
Short range order in Ge-Ga-Se glasses
}

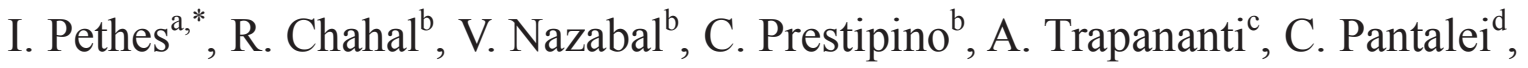 \\ B. Beuneu ${ }^{\mathrm{d}}$, B. Bureau ${ }^{\mathrm{b}}$, P. Jóvári ${ }^{\mathrm{a}}$ \\ ${ }^{a}$ Wigner Research Centre for Physics, Hungarian Academy of Sciences, H-1525 Budapest, POB 49, \\ Hungary \\ ${ }^{\mathrm{b}}$ Institut Sciences Chimiques de Rennes, UMR-CNRS 6226, Campus de Beaulieu, Université de \\ Rennes 1, 35042 Rennes Cedex, France \\ ${ }^{c}$ CNR-IOM-OGG c/o ESRF, F-38043 Grenoble, France \\ ${ }^{\mathrm{d}}$ Laboratoire Léon Brillouin, CEA-Saclay 91191 Gif sur Yvette Cedex, France
}

\begin{abstract}
Short range order of glassy $\mathrm{Ge}_{20} \mathrm{Ga}_{10} \mathrm{Se}_{70}$ and $\mathrm{Ge}_{20} \mathrm{Ga}_{5} \mathrm{Se}_{75}$ was investigated by neutron diffraction and extended X-ray absorption fine structure spectroscopy (EXAFS) at Ge, Ga and Se K-edges. For each composition large scale structural models were obtained by fitting simultaneously the four experimental datasets in the framework of the reverse Monte Carlo simulation technique. It was found that both $\mathrm{Ge}$ and $\mathrm{Ga}$ are predominantly fourfold coordinated. The quality of the fits was strongly improved by introducing Ge-Ga bonding. Models giving the best agreement with experimental data show that Ga has a complex effect on the Ge-Se host matrix: i) it enters the covalent network by forming Ga-Ge bonds ii) by decreasing the number of Se atoms around Ge, it contributes to the formation of Se-Se bonds, which may explain the higher solubility of lanthanide ions iii) the average coordination number of Se increases due to the Ga-Se 'extra' bonds. The higher average coordination of the network may be responsible for the increase of $T_{\mathrm{g}}$ upon adding $\mathrm{Ga}$ to Ge-Se glasses.
\end{abstract}

Corresponding author. E-mail address: pethes.ildiko@wigner.mta.hu 


\section{Introduction}

Chalcogenide glasses possess noteworthy physical properties, such as low phonon energy, optical nonlinearity several orders of magnitude greater than that of silica glass, broad transmittance in the mid infrared range, high ionic conductivity, or large photosensitivity [1-3]. These properties make them prominent candidates for technical applications in telecommunication, optoelectronics, photonics or energy storage [4-6].

Chalcogenide glasses can be described as covalent networks with average connectivity controlled by the coordination number of the participant elements. It has been found that in glasses of the groups 14, 15 and 16 (e.g. Ge-As-Se [7], Ge-As-Te [8], Ge-Sb-Te [9]) all components follow the 8-N rule [10], but group 13 elements, such as gallium or indium can be fourfold coordinated as well. Moreover, according to some recent publications on chalcogenide glasses, the average coordination number of chalcogenide atoms can also deviate from the 8-N rule in the presence of metallic components [11].

Several studies on Ga-Ge-Se glassy system agree that both Ge and Ga atoms are fourfold coordinated $[12,13]$. In the chemically ordered network model, Ge-Ga-Se glasses can be pictured to be made up of corner- and edge-shared tetrahedra. The Ga-Se and Ge-Se heteronuclear bonds are prevalent, Se-Se bonds appear only in Se rich compositions while metal-metal $(\mathrm{Ge} / \mathrm{Ga}-\mathrm{Ge} / \mathrm{Ga})$ bonds can be found in Se-deficient glasses only [13-15]. The average coordination number of Se-atoms is controversial. 2-fold coordinated Se atoms are reported on the basis of Raman, extended X-ray absorption fine structure and X-ray photoelectron spectroscopy [15], but Raman and multinuclear solid state nuclear magnetic resonance spectroscopy investigations showed the formation of triply coordinated Se atoms [12], similarly to crystalline $\mathrm{Ga}_{2} \mathrm{Se}_{3}$ [16] and GaSe [17]. To explain the physical properties of these glasses a previous model assumed 3-fold coordinated Se atoms and 6-fold coordinated Ga atoms [18].

Similarly in Ga-Ge-S glasses, it has been found that $\mathrm{Ge}$ and $\mathrm{Ga}$ are 4-fold coordinated, $\mathrm{GaS}_{4 / 2}$ and $\mathrm{GeS}_{4 / 2}$ tetrahedral units are cross-linked via bridging sulfur atoms [19,20]. A small number of Ge-Ge and $\mathrm{S}-\mathrm{S}$ bonds were reported in $\mathrm{Ge}_{25}(\mathrm{As}, \mathrm{Ga})_{10} \mathrm{~S}_{65}$ glasses, possessing some chemical disorder [21]. Formation of $\mathrm{GaS}_{4 / 2}$ tetrahedra from $\mathrm{Ga}_{2} \mathrm{~S}_{3}$ necessarily brings about the formation of metal-metal bonds that can either be Ge-Ge or Ga-Ga [22-24]. We also note here that threefold coordinated S has been suggested by a recent Raman scattering study on $0.8 \mathrm{GeS}_{2}-0.2 \mathrm{Ga}_{2} \mathrm{~S}_{3}[25]$.

Not only gallium containing chalcogenide glasses deviate from the $8-\mathrm{N}$ rule. The investigation of Ge- 
In-Se system using X-ray diffraction, neutron diffraction and EXAFS measurements together with reverse Monte Carlo simulation method showed that the coordination number of In is around 3.3-3.5 while that of Se increases with increasing In content and reaches $\sim 2.5$ for $\mathrm{Ge}_{17} \mathrm{In}_{15} \mathrm{Se}_{68}$ [11]. In this context, it appeared interesting to explore glass richer in selenium, in particular to investigate the coordination of Se and $\mathrm{Ga}$ and the existence of homopolar bonds. Two glasses were selected due to their technological interest for photonics applications: $\mathrm{Ge}_{20} \mathrm{Ga}_{10} \mathrm{Se}_{70}$ and $\mathrm{Ge}_{20} \mathrm{Ga}_{5} \mathrm{Se}_{75}$. Their structure was investigated by neutron diffraction and EXAFS. Though the neutron scattering lengths of the components are rather close to each other (Ge: $8.185 \mathrm{fm}$, Ga: $7.288 \mathrm{fm}$, Se: $7.97 \mathrm{fm}$ ) neutron diffraction data are still useful due to their higher accuracy of absolute normalization that permits the determination of (neutron weighted) coordination numbers with a low uncertainty. This is especially useful if the aim is the accurate determination of the coordination number of the main component. Large scale models were generated by fitting the experimental datasets simultaneously by the reverse Monte Carlo simulation technique (RMC) [26,27]. Short range order parameters were obtained by the analysis of particle configurations.

\section{Experimental}

Ge-Ga-Se glasses were prepared by conventional melt quenching method. Ga, Ge and Se with high purity $(\geq 5 \mathrm{~N})$ were used. Chemical reagents were put in silica tubes and pumped under vacuum $\left(10^{-4}\right.$ mbar) for $20 \mathrm{~h}$. After first sealing, Se was distilled to remove impurities like $\mathrm{OH}, \mathrm{H}_{2} \mathrm{O}$ and Se-H. After distillation of Se, the ampoule was sealed and put in a rocking furnace for melting at $850{ }^{\circ} \mathrm{C}$ during $8 \mathrm{~h}$. The ampoule was quenched into water, followed by annealing at $T_{\mathrm{g}}-20^{\circ} \mathrm{C}$ for $3 \mathrm{~h}$. DSC measurements were performed with $10 \mathrm{mg}$ powdered samples, heated up to $450{ }^{\circ} \mathrm{C}$ at heating rate of $10{ }^{\circ} \mathrm{C} / \mathrm{min}$.

The composition of each sample was checked by using scanning electron microscopy with an energydispersive X-ray analyzer (SDD X-Max 80mm2 Oxford Instruments AZtecEnergy ) at $20 \mathrm{kV}$. The real chemical composition of glass samples $\left(\mathrm{Ge}_{21.1} \mathrm{Ga}_{10.3} \mathrm{Se}_{68.6}\right.$ and $\left.\mathrm{Ge}_{21} \mathrm{Ga}_{5} \mathrm{Se}_{74}\right)$ is in agreement with the nominal composition taking into account the error limit of the EDX method used ( \pm 1 at.\%). Density of glass was determined by using a Mettler Toledo XS64. A $3 \mathrm{~mm}$ sample was put in the analytical balance and the density is determined by averaging three measurements.

Neutron diffraction experiments were carried out at the 7C2 diffractometer (LLB, Saclay). The wavelength of the incident radiation was $0.72 \AA$. Powdered samples were filled into vanadium sample 
holders with $6 \mathrm{~mm}$ diameter and $0.1 \mathrm{~mm}$ wall thickness. Scattered intensities were corrected for detector efficiency, background scattering, absorption and multiple scattering following standard procedures.

EXAFS spectra were collected at GILDA-BM08 beamline of the ESRF (Grenoble, France) at the Ga, $\mathrm{Ge}$ and $\mathrm{Se}$ K-edges. The beam was monochromatized by using a fixed-exit double crystal monochromator equipped with Si (311) crystals and operated with flat crystals. Two Pd-coated mirrors working at an incidence angle of $3.6 \mathrm{mrad}$ were used for harmonics rejection. Data were recorded in transmission mode using ionization chambers filled with Ar gas at different pressures to achieve the optimal efficiency in the working energy ranges $(10 \%, 80 \%$ and $90 \%$ of absorption for the I0, I1 and IR ionization chambers). Glassy samples were finely ground, mixed with cellulose powder and pressed into pellets. The amount of sample in each pellet was chosen considering the glass composition to give an absorption $\mu \mathrm{t} \sim 1.5$ just above the selected absorption edge.

The extraction of the $\chi(k)$ EXAFS signals was performed by using the VIPER code [28]. Raw $\chi(k)$ data (see Figure 1) were filtered in two steps: first $k^{3} \chi(k)$ was forward Fourier-transformed into $r$-space using a Kaiser-Bessel window $(\alpha=1.5)$. The $k$-range of transformation was $1.85 \AA^{-1}-13.3 \AA^{-1}$ for the Ga edge and $1.85 \AA^{-1}-16 \AA^{-1}$ for the Ge and Se edges. The resulting $r$-space data were backtransformed using a rectangular window (usually over the $r$-space range $1.1 \AA$ - $2.8 \AA$ ).

Raman scattering spectra were measured at room-temperature by a HR800 (Horiba Jobin-Yvon) type Raman spectrophotometer with $785 \mathrm{~nm}$ laser diode as excitation source for $10 \mathrm{~s}$, averaging 10 accumulations. Light intensity of laser beam on the sample was kept at low level to avoid alterations of Raman spectra due to photoinduced structural changes. The position of the $520 \mathrm{~cm}^{-1}$ band of Si was examined at beginning and end of the experiment in order to control any possible band shifts.

\section{Reverse Monte Carlo simulation}

Glass compositions, densities and the datasets fitted by $\mathrm{RMC}++$ code [27] are given in Table 1. Simulation boxes contained 20000 atoms. Several models were investigated with different combinations of allowed bonds. In order to avoid unphysically short interatomic distances for nearest neighbors, a series of minimum accepted interatomic distances (cut offs), usually 2.1-2.2 $\AA$, were applied in the simulations. Such cut offs were also used to preclude specific bonds, in such case the corresponding cut off has been increased to $2.9 \AA$ for the couple of atoms involved in the forbidden 
pairs.

EXAFS backscattering factors (merging backscattering phases, amplitudes and $\mathrm{S}_{0}{ }^{2}$ values) were obtained by the feff 8.4 code [29]. For details of fitting EXAFS and diffraction datasets with RMC we refer to the literature [27].

Calculations were carried out according to the following protocol:

1. Initial configurations were obtained by placing atoms at random in the simulation boxes and moving them around to satisfy cut off constraints. In the next step 'floating atoms' (with zero neighbors) and unreasonably high total coordination numbers (e.g. 4 or higher for Se) were also eliminated but no target coordination number values were given as constraints.

All simulations were started from the as-obtained configurations. The number of accepted moves was usually around 1-2 times $10^{7}$.

2. Coordination constraint-free runs were carried out to see which types of bonds are needed to get reasonable fits of the experimental data. In these calculations, certain bonds were forbidden by raising the corresponding cut-offs to $2.9 \AA$ but the total coordination numbers were not constrained. At first a reference model was obtained by carrying out a simulation in which all bond types were allowed. Then Ga-Ga, Ge-Ge and Ge-Ga bonds were forbidden in various combinations. The obtained models were assessed by their cumulative relative R-factors. The relative R-factor of a model with respect to the reference model is defined by the following equation:

$$
R_{r}=\frac{\sqrt{\sum\left(S_{\bmod }-S_{\text {exp }}\right)^{2}}}{\sqrt{\sum\left(S_{\bmod }^{r e f}-S_{\text {exp }}\right)^{2}}}
$$

where $S_{\text {mod }}$ and $S_{\text {exp }}$ are the model and experimental curves structure factors (or EXAFS curves), $S_{\text {mod }}^{\text {ref }}$ is the structure factor (or EXAFS curves) of the reference model, and the summation runs over the experimental points. Cumulative relative R-factors $\left(R_{\mathrm{c}}\right)$ are obtained by simply averaging the relative $\mathrm{R}$-factors of a model.

3. Finally coordination constraints were applied to check the fulfilment of the $8-\mathrm{N}$ rule and other assumptions from the literature. In these models $\mathrm{Ge}$ and/or $\mathrm{Ga}$ atoms were forced to have four neighbors (any allowed type) while the coordination number of Se was either allowed to change freely or was forced to be 2 . The coordination constraints were fulfilled by at least $95 \%$ of the atoms. 


\section{Results and discussion}

\subsection{RMC investigations}

$\mathrm{Ge}_{20} \mathrm{Ga}_{10} \mathrm{Se}_{70}$

A long standing question of the short range order of Ge-Ga-Se glasses is whether metal-metal bonds exist or Ge and Ga atoms bind only to Se. To answer this question models with every possible combination of allowed metal-metal bonds (including the model where all metal-metal bonds are forbidden) were tested. It was found that if all metal-metal bonds are forbidden then the relative Rfactors of both the Ge and Ga EXAFS measurements increased strongly. As the measurements can still be fitted properly if Ge-Ge and Ga-Ga bonds are forbidden but Ge-Ga pairs are allowed these runs suggest that the necessary metal-metal bond is most probably the Ge-Ga one.

The coordination constraint-free reference model gives an average coordination number 3.84 for germanium and 3.8 for gallium, confirming that $\mathrm{Ge}$ and $\mathrm{Ga}$ atoms are predominantly fourfold coordinated. In fact, forcing Ge and Ga atoms to be fourfold coordinated does not change the overall fit quality. The strictest model where we still got adequate fits was the one in which Ge-Ga bonds were allowed, Ga-Ga and Ge-Ge bonds were eliminated, and both Ge and Ga were forced to be fourfold coordinated. Hereafter this will be called as 'final model'. The simulated diffraction and EXAFS curves from this final model are compared to the experimental data in Figures 2 and 3. The partial pair correlation functions obtained by this model are presented in Figure 4. The coordination numbers and the bond distances obtained with the final model are in Table 2.

The uncertainty of Ge-Ga coordination number was estimated by forcing it stepwise to lower or higher values and monitoring the changes in the R-factors [7]. The lower limit of the Ge-Ga coordination number according to this procedure is $\sim 0.45$. If we accept that both Ge and Ga are strictly fourfold coordinated, then the existence of such a lower limit for the Ge-Ga coordination number involves the existence of strict upper limits for the Ge-Se and Ga-Se coordination numbers $(\sim 3.5$ and $~ 3.1$, respectively).

The formation of Ge-Ga bonds also means the decrease of the number of Se-Ge and Se-Ga bonds and thus promotes Se-Se bonding even in stoichiometric compositions. It is reasonable to assume that the increased solubility of rare earths is connected to the presence of small Se clusters that can host large cations.

The total average coordination number of selenium $\left(N_{\mathrm{Se}}\right)$ proved to be higher than two regardless the constraints applied to the type and number of the neighbors of $\mathrm{Ge}$ and $\mathrm{Ga}$. $N_{\mathrm{Se}}$ always increases if metal-metal bonds are forbidden. Different coordination constraints were applied to selenium to check 
whether the deviation from the 8-N rule is significant. We found that it was not possible to get reasonable fits and reasonable structural parameters simultaneously with twofold coordinated Se atoms. Such models either gave significantly poorer fits or exhibit some spurious features to compensate the forced decrease of the Se coordination number (e.g. the coordination number of Ge becomes as high as 4.6).

We also made simulation runs with constraints for the average coordination number of selenium (increasing and decreasing it from 2.3, the value obtained in the final model). Figure 5 shows the relative R-factors of the neutron diffraction dataset $\left(R_{\mathrm{r}}^{\mathrm{ND}}\right)$ at different $N_{\mathrm{Se}}$ values. The fit quality decays drastically for $N_{\mathrm{Se}}<2.15$. From these simulation runs, it can be concluded that the average coordination number of selenium is around $2.3 \pm 0.15$.

It is remarkable that in the final model (4-fold coordinated $\mathrm{Ge}$ and $\mathrm{Ga}, \mathrm{Ge}-\mathrm{Ge}$ and $\mathrm{Ga}-\mathrm{Ga}$ bonds are forbidden) the sum of Se-Se and Se-Ge coordination numbers is very close to two (1.97) suggesting that Se forms the 'third bond' with Ga. It is not so surprising if we consider that glass-ceramics formed from Ge-Ga-Se glasses will mainly present $\mathrm{Ga}_{2} \mathrm{Se}_{3}$ crystallites [30]; the $\mathrm{Ga}_{2} \mathrm{Se}_{3}$ crystallized phase is built up from corner sharing $\mathrm{GaSe}_{4}$ tetrahedra and one third of Se is three-fold coordinated in these tetrahedra. A similarly increased coordination number of Te was reported previously in the Ga-Ge-Te system [31].

\section{$\mathrm{Ge}_{20} \mathrm{Ga}_{5} \mathrm{Se}_{75}$}

Due to the lower gallium concentration, the uncertainty of Ga-related structural parameters is higher here than in case of $\mathrm{Ge}_{20} \mathrm{Ga}_{10} \mathrm{Se}_{70}$. However, it is reasonable to suppose that Ga behaves essentially in the same way in the two compositions. Therefore the coordination number of gallium was forced to be four in each simulation run but the scenario applied for $\mathrm{Ge}_{20} \mathrm{Ga}_{10} \mathrm{Se}_{70}$ was used otherwise (e.g. test of $\mathrm{Ge} / \mathrm{Ga}-\mathrm{Ge} / \mathrm{Ga}$ bonds, test of Se total average coordination number). In accordance with the lower Ga (and higher Se) content it has been found that neither Ga-Ga nor Ge-Ge bonds improve the fit quality. On the other hand, elimination of all metal-metal bonding results in a significant worsening of fits. Coordination numbers and bond lengths are shown in Table 3. Our results suggest that the environment of gallium atoms does not strongly differ in the two compositions. The total coordination number of Se is significantly higher than $2(2.25 \pm 0.15)$.

Introducing $\mathrm{Ga}$ at the expense of $\mathrm{Se}$ in $\mathrm{Ge}_{20} \mathrm{Se}_{80}$ parent glass brings about the increase of mean coordination number, and thus enhances the stability of the vitreous state. This is manifested by higher values of the glass transition temperature (see Table 4). 


\subsection{Comparison with Raman spectroscopy analysis}

The glass network structure can be easily affected by the synthesis conditions and slight changes in composition. Nevertheless, the Raman spectra of $\mathrm{Ge}_{20} \mathrm{Se}_{80}, \mathrm{Ge}_{20} \mathrm{Ga}_{5} \mathrm{Se}_{75}$ and $\mathrm{Ge}_{20} \mathrm{Ga}_{10} \mathrm{Se}_{70}$ glasses are, as expected dominated by two broad bands in the $\sim 175-225 \mathrm{~cm}^{-1}$ and $\sim 230-325 \mathrm{~cm}^{-1}$ spectral regions (see Figure 6). The two peaks observed at $\sim 200$ and $\sim 215 \mathrm{~cm}^{-1}$ are attributed to $A_{1}$ symmetric stretching mode of corner linked $\mathrm{GeSe}_{4 / 2}$ tetrahedra and to $\mathrm{A}_{1}{ }^{\mathrm{c}}$ breathing vibration mode (also called companion mode) likely related to symmetric vibration mode of $\mathrm{GeSe}_{4 / 2}$ tetrahedra connected by edge [32-34]. The bands related to germanium and gallium structural units certainly overlap due to close atomic weights of these elements and amorphous character of the matrix leading to broad vibration modes [18]. With introduction of $\mathrm{Ga}$, the maximum of the dominant band of Raman spectra slightly shifts to higher frequency from $195 \mathrm{~cm}^{-1}$ to $197 \mathrm{~cm}^{-1}$. A slight broadening of the peak width is observed when Ga concentration increases. One can also note that the ratio between the intensity of the $A_{1}$ and the $A_{1}{ }^{c}$ contributions is slightly decreasing, suggesting that corner linked tetrahedra are broken first by Ga introduction.

The broad band of lower intensity, covering the $\sim 230-325 \mathrm{~cm}^{-1}$ region possess several contributions. The asymmetry of the band peaking at $265 \mathrm{~cm}^{-1}$ reveals the presence of homonuclear Se-Se bonds originating from different kind of entities : i) Se chains at $235 \mathrm{~cm}^{-1}[13,33,35]$ also proposed at 265 $\mathrm{cm}^{-1}[13,36,37]$, ii) stretching mode of Se-Se bond in $\mathrm{Se}_{8}$ rings $[33,35]$ and at the outrigger raft structure at $\sim 245-250 \mathrm{~cm}^{-1}$ [35], iii) $\mathrm{Ge}(\mathrm{Ga}) \mathrm{Se}_{4 / 2}$ corner-shared dimers linked by dimers or small chains at $\sim 265$ $\mathrm{cm}^{-1}[13,38]$. A weak contribution of $\mathrm{F}_{2}$ asymmetric vibration mode of $\mathrm{Ge}(\mathrm{Ga}) \mathrm{Se}_{4 / 2}$ tetrahedral at higher frequencies $\left(\sim 285-315 \mathrm{~cm}^{-1}\right)$ is not affected by Ga increase as the main bands at $199 \mathrm{~cm}^{-1}$ in term of intensity. The decrease of $265 \mathrm{~cm}^{-1}$ band with increasing Ga content is representative of the decrease of Se content compared to $(\mathrm{Ga}+\mathrm{Ge})$ concentration.

The observed band in the range of $166 \mathrm{~cm}^{-1}-175 \mathrm{~cm}^{-1}$ is usually associated with metal-metal bonds (e.g. $\mathrm{Ge}(\mathrm{Ga})-\mathrm{Ge}(\mathrm{Ga})$ bond vibrations in $\mathrm{Ge}(\mathrm{Ga})_{2} \mathrm{Se}_{6 / 2}$ ethane like entities) $[13,18,33,34,36,38]$. According to our RMC results, we can clearly conclude that this band increasing with Ga content reveals the presence of Ge-Ga bonds in Se-rich Ge-Ga-Se glasses.

\section{Conclusions}


The structure of $\mathrm{Ge}_{20} \mathrm{Ga}_{10} \mathrm{Se}_{70}$ and $\mathrm{Ge}_{20} \mathrm{Ga}_{5} \mathrm{Se}_{75}$ glasses was investigated by neutron diffraction and EXAFS spectroscopy. Experimental datasets were fitted simultaneously by the reverse Monte Carlo simulation technique. Several models were created by eliminating various bond types and using coordination constraints. It has been established that $\mathrm{Ge}$ and $\mathrm{Ga}$ are both fourfold coordinated. Our results also show that $\mathrm{Ga}$ enters the germanium selenide host network by forming $\mathrm{Ge}-\mathrm{Ga}$ bonds. By decreasing the number of Se atoms around Ge and Ga these bonds contribute to the formation of Se-Se bonds. Thus, adding Ga to the Ge-Se matrix brings about the increase of 'free' Se atoms which may higher solubility of rare earths in Ge-Ga-Se glasses. The average coordination number of Se increases due to the Ga-Se 'extra' bonds. The higher average coordination of the network is responsible for the increase of $T_{\mathrm{g}}$ upon adding $\mathrm{Ga}$ to $\mathrm{Ge}_{20} \mathrm{Se}_{80}$ glasses.

\section{Acknowledgments}

V. Nazabal, B. Bureau and R. Chahal are thankful to ADEME, the French Environment and Energy Management Agency for financial support. I. Pethes and P. Jóvári were supported by OTKA (Hungarian Basic research Fund) Grant No. 083529.

\section{References}

[1] A. Zakery, S.R. Elliott, J. Non-Cryst. Solids 330 (2003) 1.

[2] K. Tanaka, J. Mater. Sci.-Mater. Electron.16, (2005) 633.

[3] J. Saienga, S.W. Martin, J. Non-Cryst. Solids 354 (2008) 1475.

[4] B.J. Eggleton, B. Luther-Davies, K. Richardson, Nature Photonics 5 (2011) 141.

[5] X.H. Zhang, B. Bureau, P. Lucas, C. Boussard-Pledel, J. Lucas, Chem.-Eur. J. 14 (2008) 432.

[6] J.S. Sanghera, I.D. Aggarwal, J. Non-Cryst. Solids 256 (1999) 6.

[7] I. Pethes, I. Kaban, R.P. Wang, B. Luther-Davies, P. Jóvári, J. Alloy. Compd. 623 (2015) 454.

[8] P.E. Lippens, J.C. Jumas, J. Olivier-Fourcade, L. Aldon, J. Non-Cryst. Solids 271 (2000) 119.

[9] P. Jóvári, I. Kaban, J. Steiner, B. Beuneu, A. Schöps, A. Webb, Phys. Rev. B 77 (2008) 035202. 
[10] N. Mott Adv. Phys. 16 (1967) 49.

[11] I. Kaban, P. Jóvári, T. Petkova, P. Petkov, A. Stoilova, W. Hoyer, B. Beuneu, J. Phys. Condensed Matter 22 (2010) 404205.

[12] A.W. Mao, B.G. Aitken, R.E. Youngman, D.C. Kaseman, S. Sen, J. Phys. Chem. B 117 (2013) 16594.

[13] P. Němec, B. Frumarová, M. Frumar, J. Non-Cryst. Solids 270 (2000) 137.

[14] S. Mahadevan, A. Giridhar, J. Non-Cryst. Solids 152 (1993) 42.

[15] R. Golovchak, L. Calvez, E. Petracovschi, B. Bureau, D. Savytskii, H. Jain, Mat. Chem. Phys. 138 (2013) 909.

[16] D. Lübbers, V. Leute, J. Solid State Chem. 43 (1982) 339.

[17] N.C. Fernelius, Prog. Cryst. Growth Charact. Mater. 28 (1994) 275.

[18] K. Maeda, T. Sakai, K. Sakai, T. Ikari, M. Munzar, D. Tonchev, S.O. Kasap, G. Lucovsky, J. Mater. Sci.-Mater. Electron. 18 (2007) S367.

[19] A. Tverjanovich, Y.S. Tveryanovich, S. Loheider, J. Non-Cryst. Solids 208 (1996) 49.

[20] J.H. Song, Y.G. Choi, J. Heo, J. Non-Cryst. Solids 352 (2006) 423.

[21] A.C. Hannon, B.G. Aitken, J. Non-Cryst. Solids 256-257 (1999) 73.

[22] C. Julien, S. Barnier, M. Massot, N. Chbani, X. Cai, A.M. Loireau-Lozac'h, M. Guittard, Mater. Sci. Eng. B 22 (1994) 191.

[23] Y. Ledemi, S.H. Messaddeq, I. Skhripachev, S.J.L. Ribeiro, Y. Messaddeq, J. Non-Cryst. Solids 355 (2009) 1884.

[24] C.G. Lin, L. Calvez, H.Z. Tao, M. Allix, A. Moreac, X.H. Zhang, X.J. Zhao, J. Solid State Chem.184 (2011) 584.

[25] P. Masselin, D. Le Coq, A. Cuisset, E. Bychkov, Opt. Mater. Express 2 (2012) 1768.

[26] R.L. McGreevy, L. Pusztai, Mol. Simulat. 1 (1988) 359.

[27] O. Gereben, P. Jóvári, L. Temleitner, L. Pusztai, J. Optoelectron. Adv. Mater. 9 (2007) 3021.

[28] K.V. Klementev, J. Phys. D: Appl. Phys. 34 (2001) 209.

[29] A.L. Ankudinov, B. Ravel, J.J. Rehr, S.D. Conradson, Phys. Rev. B 58 (1998) 7565.

[30] L. Calvez, H.L. Ma, J. Lucas, X.H. Zhang, Adv. Mater. 19 (2007) 129.

[31] P. Jóvári, I. Kaban, B. Bureau, A. Wilhelm, P. Lucas, B. Beuneu, D.A. Zajac, J. Phys.: Condens. Matter 22 (2010) 404207.

[32] O. Matsuda, K. Inoue, K. Murase, Solid State Commun. 75 (1990) 303.

[33] S. Sugai, Phys. Rev. B 35 (1987) 1345.

[34] M. Olivier et al., Opt. Mater. Express 4 (2014) 525. 
[35] G. Lucovsky, A. Mooradia, W. Taylor, G.B. Wright, R.C. Keezer, Solid State Commun. 5 (1967) 113.

[36] R. Holomb, V. Mitsa, E. Akalin, S. Akyuz, M. Sichka J. Non-Cryst. Solids, 373 (2013) 51.

[37] K. Murase, T. Fukunaga, K. Yakushiji, T. Yoshimi, I. Yunoki, J. Non-Cryst. Solids, 59-60 (1983) 883.

[38] V. Nazabal et al., Thin Solid Films 518 (2010) 4941. 


\begin{tabular}{cccc}
\hline nominal composition & $\begin{array}{c}\text { real composition } \\
( \pm 1 \%)\end{array}$ & $\begin{array}{c}\text { density }\left(\mathrm{g} / \mathrm{cm}^{3}\right) \\
( \pm 0.01)\end{array}$ & $\begin{array}{c}\text { atomic density } \\
( \pm 0.0001)\end{array}$ \\
\hline $\mathrm{Ge}_{20} \mathrm{Ga}_{10} \mathrm{Se}_{70}$ & $\mathrm{Ge}_{21} \mathrm{Ga}_{10} \mathrm{Se}_{69}$ & 4.42 & 0.0347 \\
$\mathrm{Ge}_{20} \mathrm{Ga}_{5} \mathrm{Se}_{75}$ & $\mathrm{Ge}_{21} \mathrm{Ga}_{5} \mathrm{Se}_{74}$ & 4.38 & 0.0342 \\
\hline
\end{tabular}

Table 1 Compositions modeled and their densities.

\begin{tabular}{lcc}
\hline \multicolumn{1}{c}{ bond type } & coordination number & bond distance \\
\hline Ge-Ga & $0.75(0.45-1.6)$ & $2.48 \pm 0.03$ \\
$\mathrm{Ga}-\mathrm{Ge}$ & $1.55(0.9-3.3)$ & $2.48 \pm 0.03$ \\
$\mathrm{Ge}-\mathrm{Se}$ & $3.2(2.4-3.5)$ & $2.36 \pm 0.01$ \\
$\mathrm{Se}-\mathrm{Ge}$ & $1.0(0.75-1.1)$ & $2.36 \pm 0.01$ \\
$\mathrm{Ga}-\mathrm{Se}$ & $2.45(0.85-3.1)$ & $2.38 \pm 0.02$ \\
$\mathrm{Se}-\mathrm{Ga}$ & $0.35(0.12-0.45)$ & $2.38 \pm 0.02$ \\
$\mathrm{Se}-\mathrm{Se}$ & $0.97(0.8-1.45)$ & $2.36 \pm 0.02$ \\
\hline
\end{tabular}

Table 2 Coordination numbers and bond distances of $\mathrm{Ge}_{20} \mathrm{Ga}_{10} \mathrm{Se}_{70}$ obtained by the final model. (The values in brackets denote the range of the parameters in which the R-factors of the corresponding models are still low. The ranges are rather broad but can be most likely narrowed if changes of bond distances are also monitored. Stretched coordination numbers are often accompanied with stretched artificially short/long- bond lengths.)

\begin{tabular}{ccc}
\hline bond type & coordination number & bond distance \\
\hline Ge-Ga & 0.45 & $2.48 \pm 0.03$ \\
Ga-Ge & 1.75 & $2.48 \pm 0.03$ \\
Ge-Se & 3.6 & $2.36 \pm 0.01$ \\
Se-Ge & 1.0 & $2.36 \pm 0.01$ \\
Ga-Se & 2.25 & $2.37 \pm 0.02$ \\
Se-Ga & 0.15 & $2.37 \pm 0.02$ \\
Se-Se & 1.1 & $2.35 \pm 0.01$ \\
\hline
\end{tabular}

Table 3 Coordination numbers and bond distances of $\mathrm{Ge}_{20} \mathrm{Ga}_{5} \mathrm{Se}_{75}$ sample 


\begin{tabular}{cc}
\hline composition & $T_{\mathrm{g}}\left({ }^{\circ} \mathrm{C}\right)\left( \pm 5^{\circ} \mathrm{C}\right)$ \\
\hline $\mathrm{Ge}_{20} \mathrm{Se}_{80}$ & 162 \\
$\mathrm{Ge}_{20} \mathrm{Ga}_{5} \mathrm{Se}_{75}$ & 197 \\
$\mathrm{Ge}_{20} \mathrm{Ga}_{10} \mathrm{Se}_{70}$ & 257 \\
\hline
\end{tabular}

Table 4 Glass transition temperatures $\left(T_{\mathrm{g}}\right)$ of the investigated compositions and the parent $\mathrm{Ge}_{20} \mathrm{Se}_{80}$ glass.

\section{Figure Captions}

Figure 1 Experimental $k^{3}$ weighted Ge, Ga and Se K-edge EXAFS curves and the magnitude of their Fourier transforms (FT): $\mathrm{Ge}_{20} \mathrm{Ga}_{5} \mathrm{Se}_{75}$ (solid black line) and $\mathrm{Ge}_{20} \mathrm{Ga}_{10} \mathrm{Se}_{70}$ (dashed red line).

Figure 2 Neutron diffraction structure factor (symbols) and fit (line) for the final model (see text) of $\mathrm{Ge}_{20} \mathrm{Ga}_{10} \mathrm{Se}_{70}$ sample.

Figure $3 \mathrm{Ge}$, Ga and Se K-edge $k^{3} \chi$ filtered experimental curves (symbols) and fits (lines) for the final model of $\mathrm{Ge}_{20} \mathrm{Ga}_{10} \mathrm{Se}_{70}$.

Figure 4 Partial pair correlation functions for the final model of $\mathrm{Ge}_{20} \mathrm{Ga}_{10} \mathrm{Se}_{70}$ sample.

Figure 5 Relative R-factor of the neutron diffraction dataset at different values of the average coordination number of selenium. In this model Ge-Ge and Ga-Ga bonds were forbidden, and constraints were applied for the germanium and gallium atoms (to be fourfold coordinated).

Figure 6 Raman spectra of the $\mathrm{Ge}_{20} \mathrm{Se}_{80}, \mathrm{Ge}_{20} \mathrm{Ga}_{5} \mathrm{Se}_{75}$ and $\mathrm{Ge}_{20} \mathrm{Ga}_{10} \mathrm{Se}_{70}$ glasses at $785 \mathrm{~nm}$ laser excitation. All curves are normalized to the intensity of the strongest band. 

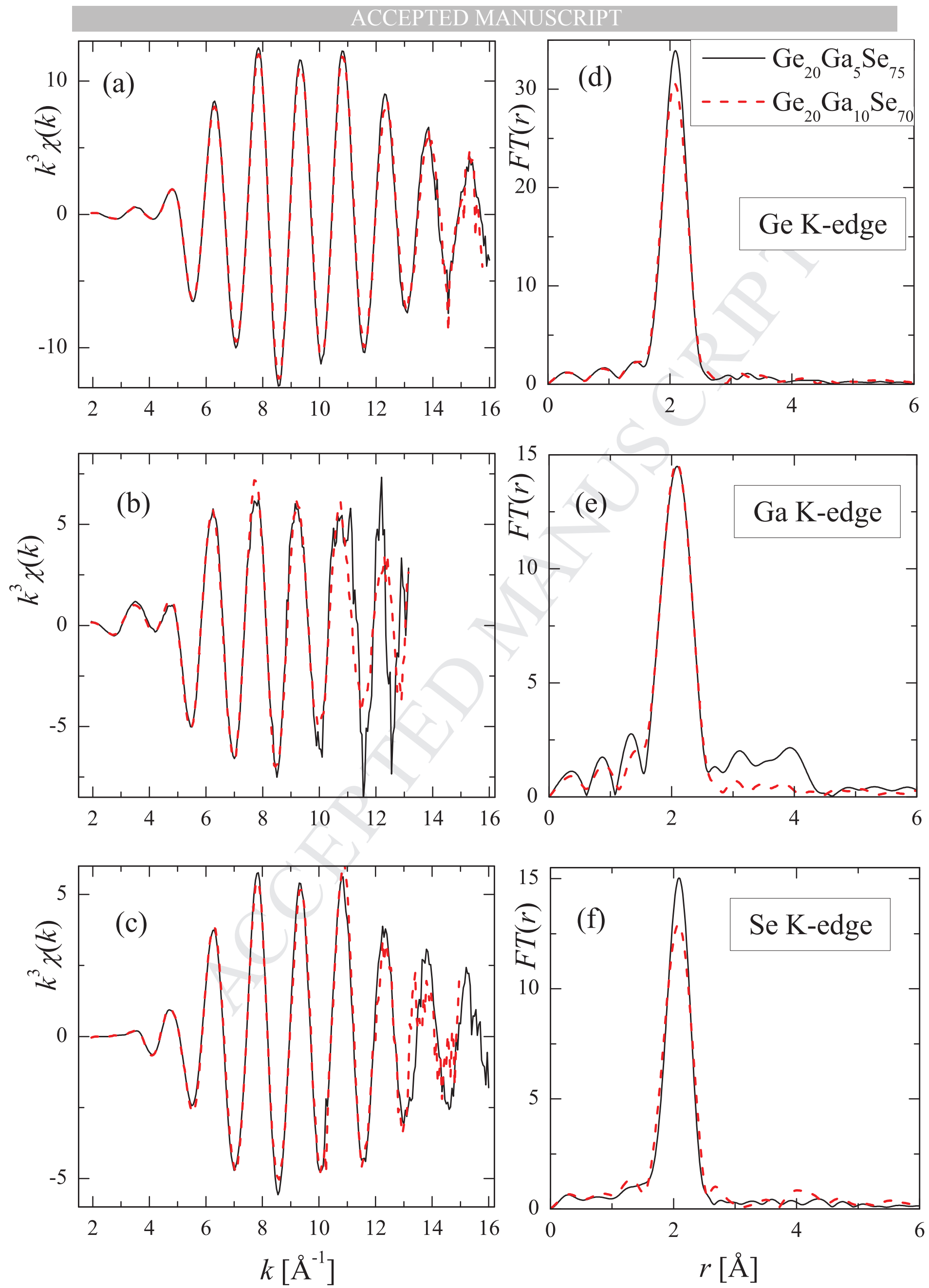


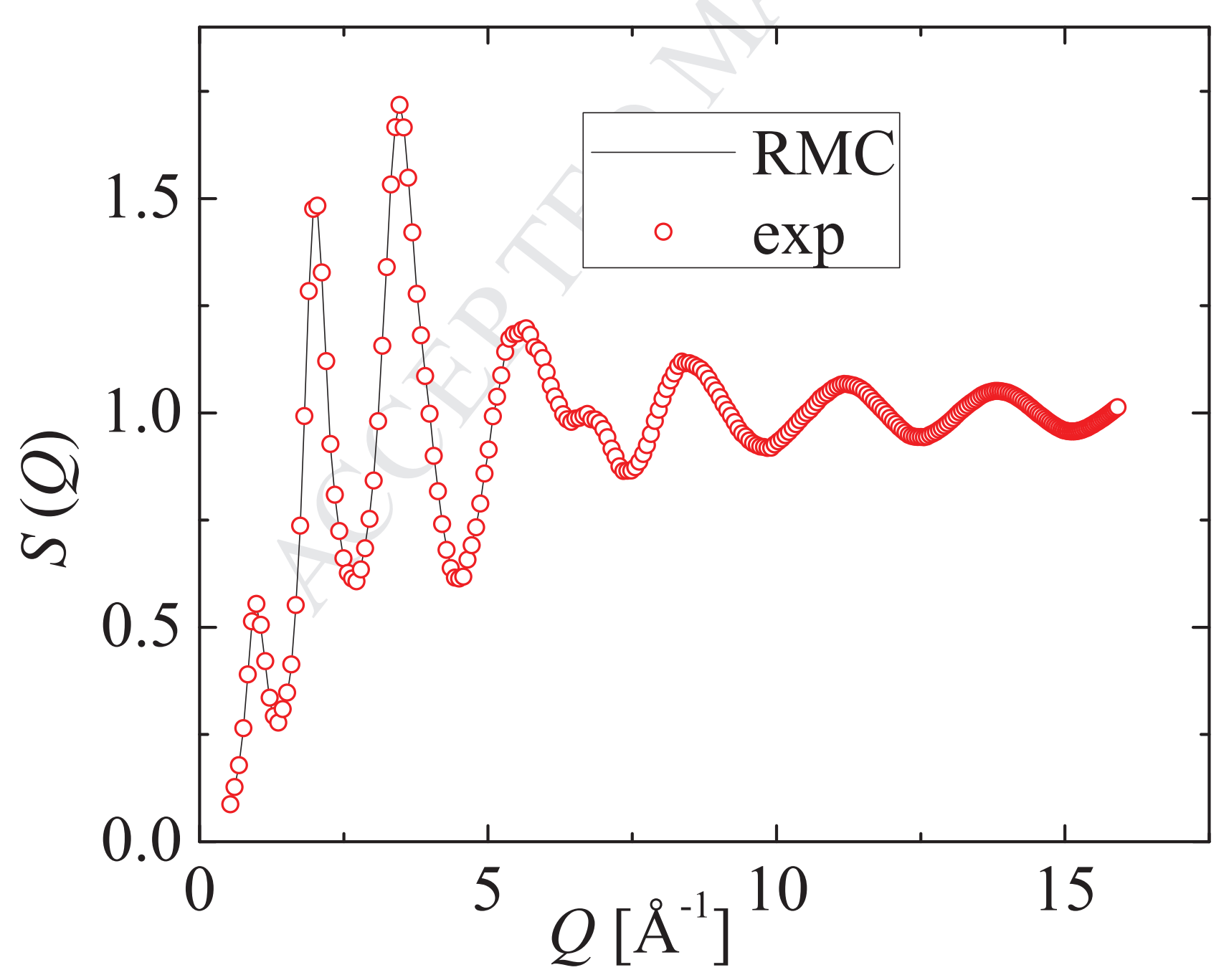




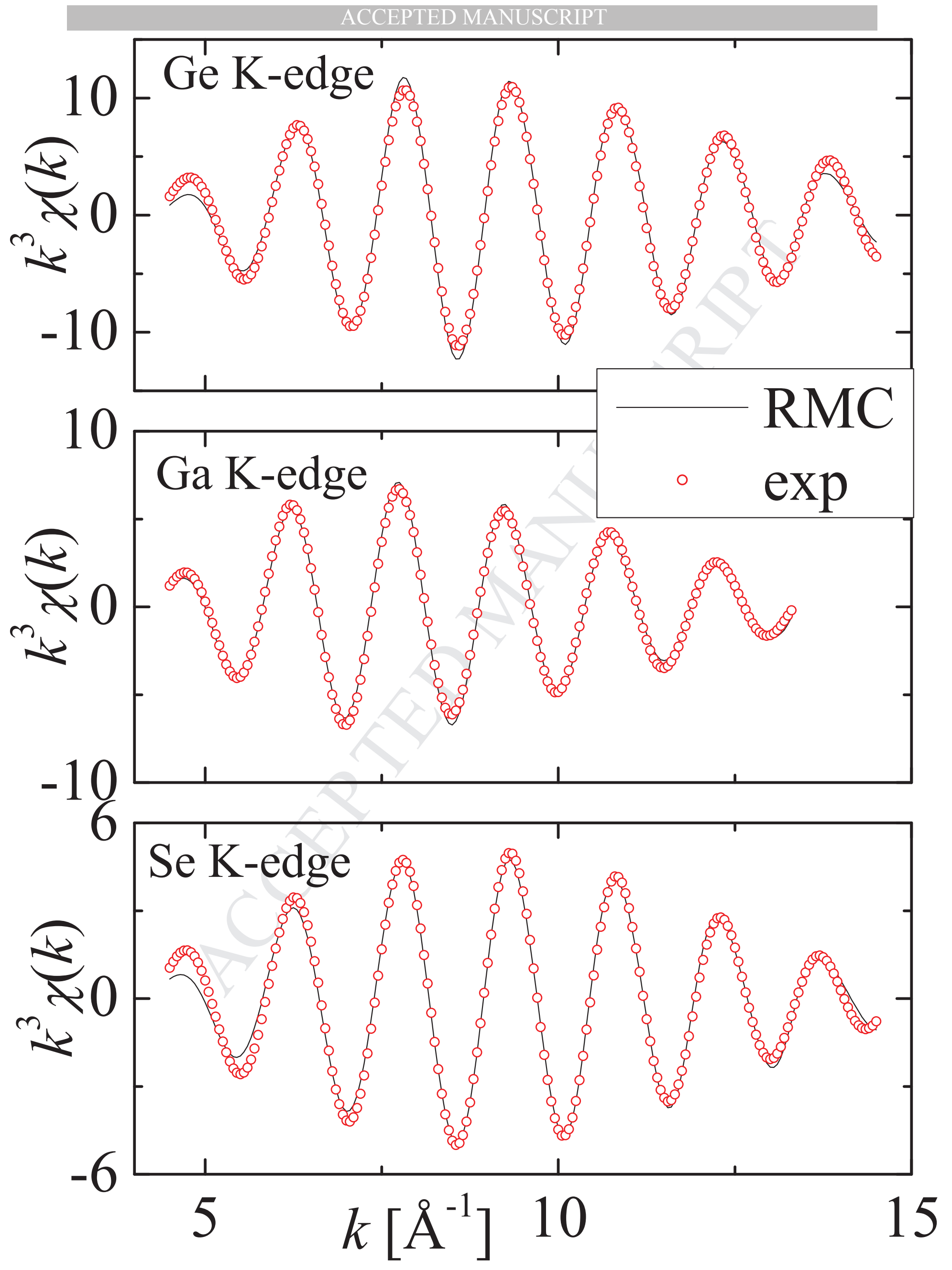



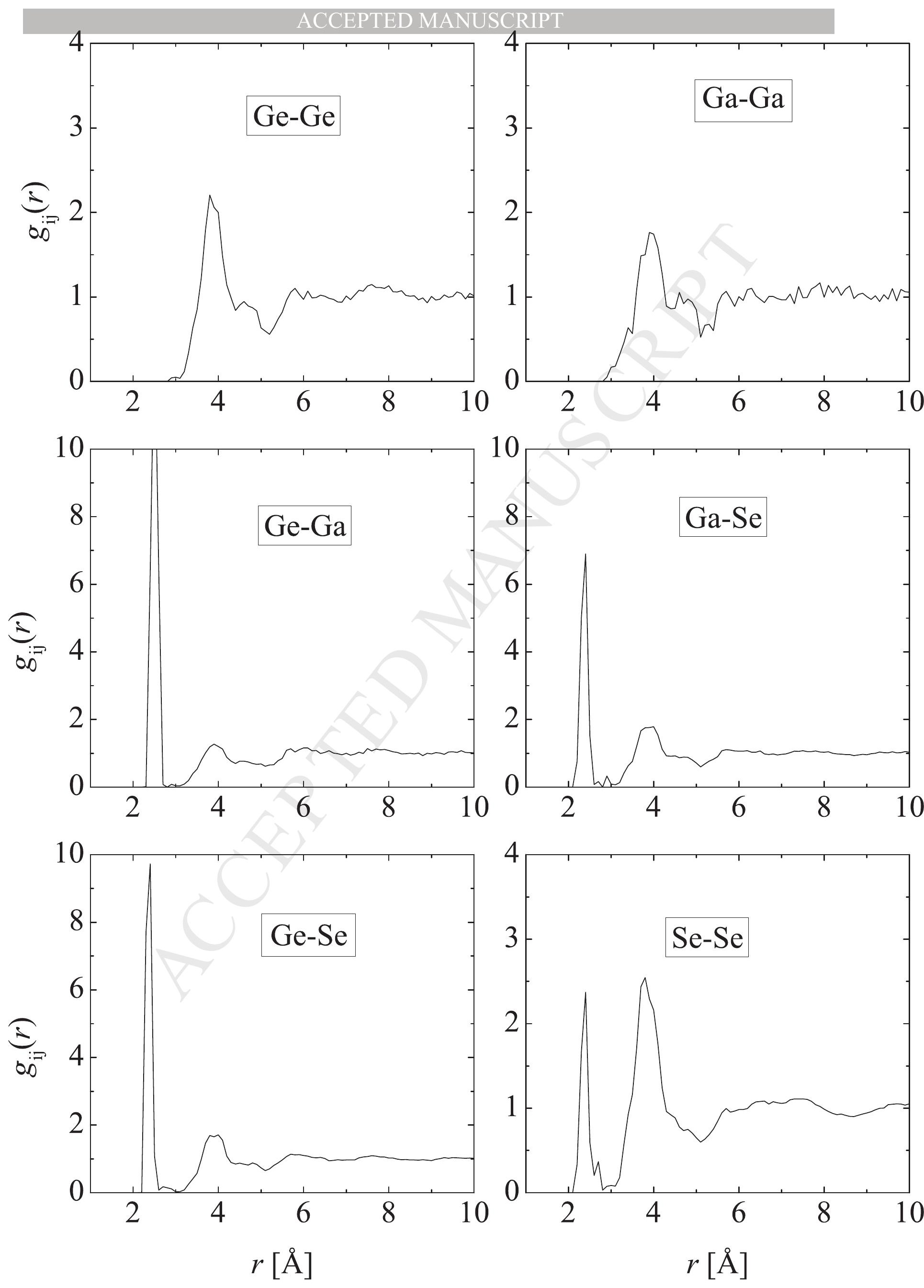


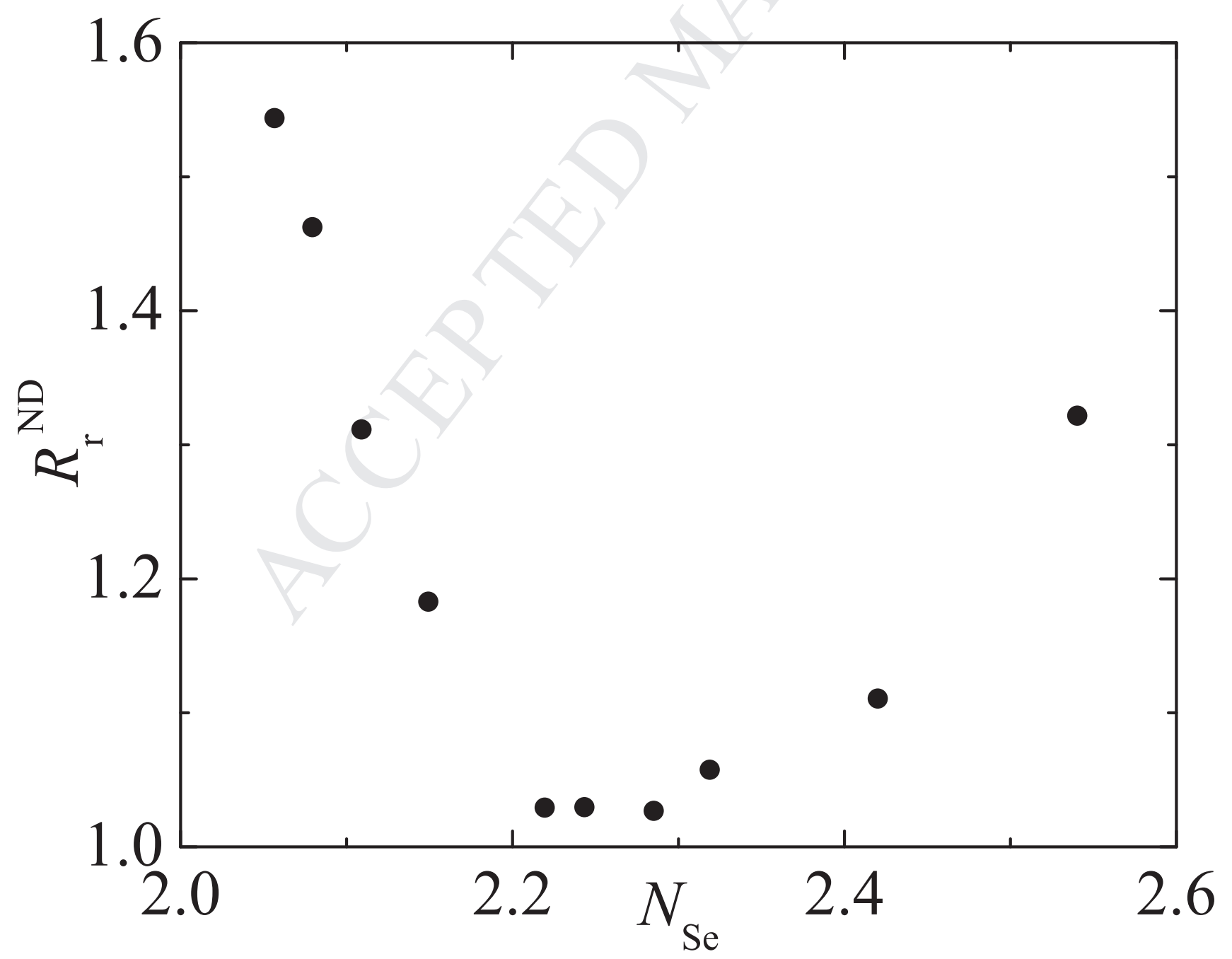




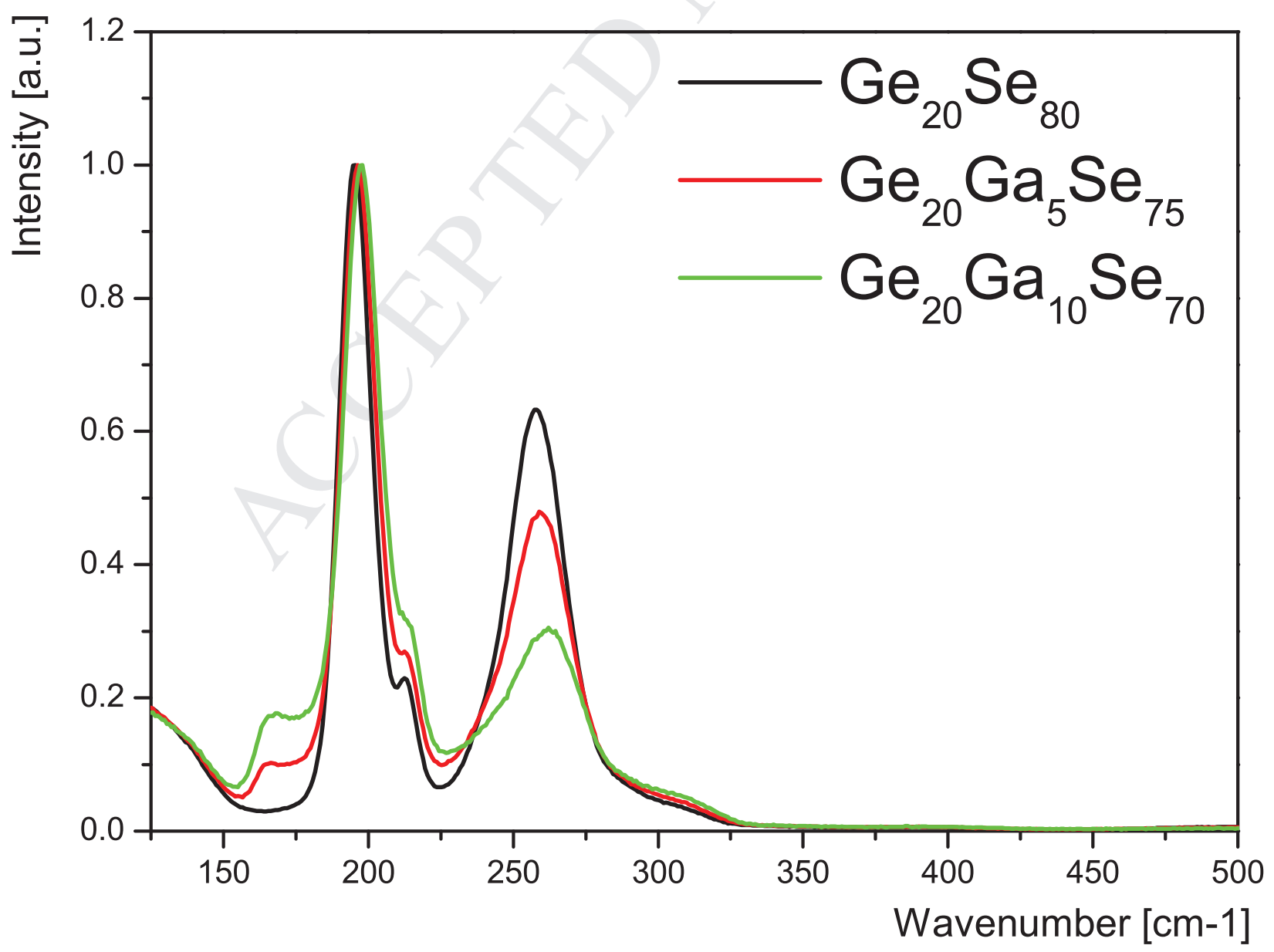


- The coordination number of Se is significantly higher than 2 .

- Ga-Ge bonds can be found even in Se-rich compositions.

- The formation of Se-Se bonds increases the solubility of rare earth ions

- The coordination number of $\mathrm{Ga}$ is 4 . 